\title{
Gastrointestinal Bleeding in Patients With Acute Respiratory Distress Syndrome: A National Database Analysis
}

\author{
Faraz Siddiqui ${ }^{a}$, Moiz Ahmed ${ }^{b}$, Saqib Abbasi ${ }^{b}$, Akshay Avula ${ }^{a}$, Abdul Hasan Siddiqui ${ }^{a}$, \\ Jobin Philipose ${ }^{\mathrm{b}, \mathrm{e}}$, Hafiz M. Khan ${ }^{\mathrm{c}}$, Tahir M. A. Khan ${ }^{\mathrm{d}}$, \\ Liliane Deeb ${ }^{\mathrm{c}}$, Michel Chalhoub
}

\begin{abstract}
Background: The goal of our study was to determine the impact of gastrointestinal bleeding (GIB) on in-hospital outcomes among acute respiratory distress syndrome (ARDS) patients, and subsequently determine the potential risk factors for the development of GIB.

Methods: ARDS patients with and without GIB were identified using the National Inpatient Sample (2002 - 2012). Linear regression analysis was used to assess impact of GIB on in-hospital mortality, length of stay and total charges. Univariate logistic regression was used to determine associated odds ratios (OR) for causes of ARDS and common comorbid conditions.
\end{abstract}

Results: We identified 149,190 ARDS patients. The incidence of GIB was the highest among patients $>60$ years $(\mathrm{P}<0.001)$. GIB was associated with longer hospitalization days ( 7.3 days versus 11.9 days, $\mathrm{P}<0.001)$, higher mortality $(11 \%$ versus $27 \%, \mathrm{P}<0.001)$ and greater economic burden $(\$ 82,812$ versus $\$ 45,951, \mathrm{P}<0.001)$. GIB was common in cirrhosis (OR: 8.3), peptic ulcer disease (OR: 3.7), coagulopathy disorders (OR: 3.003), thrombocytopenia (OR: 2.6), anemia (OR: $2.5)$ and atrial fibrillation (OR: 1.5). ARDS secondary to aspiration pneumonia (OR: 2.0), pancreatitis (OR: 2.0), sepsis (OR: 1.6) and community acquired pneumonia (OR: 0.8 ) was more likely to have GIB.

Conclusion: Our study demonstrates that GIB in ARDS patients is associated with significant increased mortality, hospitalization and health care cost.

Keywords: Gastrointestinal bleeding; ARDS; National Database

Manuscript submitted October 11, 2018, accepted November 9, 2018

aDepartment of Pulmonary \& Critical Care, Staten Island University Hospital, Northwell Health, NY, USA

bDepartment of Internal Medicine, Staten Island University Hospital, Northwell Health, NY, USA

'Department of Gastroenterology and Hepatology, Staten Island University Hospital, Northwell Health, NY, USA

dDepartment of Internal Medicine, Marshfield Clinic, WI, USA

${ }^{e}$ Corresponding Author: Jobin Philipose, 62 Newberry Avenue, Staten Island, NY 10304, USA. Email: philipose.jobin@gmail.com

doi: https://doi.org/10.14740/jocmr3660
Analysis

\section{Introduction}

Acute respiratory distress syndrome (ARDS) is a complex disorder characterized by diffuse capillary endothelial and alveolar damage. Early reports in 1978 described an increased incidence of gastrointestinal bleeding (GIB) at a rate of 11 out of 13 ARDS patients compared to 4 out of 44 mechanically ventilated chronic obstructive pulmonary disease (COPD) patients [1].

Overt GIB is a known complication ranging between $1.5 \%$ and $8.5 \%$ in critically ill patients [2-4]. In this setting, prolonged mechanical ventilation for $>48 \mathrm{~h}$ and coagulopathy are well-described risk factors for gastrointestinal bleeding [5]. ARDS appears to be an additional condition that sets the stage for developing a specific gut injury leading to bleeding. The underlying stress-related mucosal damage (SRMD) in this setting could range from asymptomatic superficial lesions and occult GIB to deeper mucosal injury and overt clinically significant hemorrhage [6]. The pathophysiology of SRMD is explained by disruption of the gastric mucosal barrier due to reduced gastric blood flow, increased reflux of bile salts and uremic toxins along with reperfusion injury $[1,7]$. SRMD is responsible for the majority of GIB in mechanically ventilated patients. It has been postulated that $75-100 \%$ of critically ill patients will have endoscopically detected stress-related mucosal erosions and subepithelial hemorrhages within $24 \mathrm{~h}$ of their admission to the critical care unit [8-10].

The current standards in the management of ARDS follows a lung-protective strategy using low tidal volumes (TVs) and increased positive end-expiratory pressures (PEEP) to prevent atelectasis and support oxygenation. This increased PEEP results in decreased venous return and cardiac output, which leads to splanchnic hypoperfusion and ischemic injury to the gastric mucosa. This condition is also associated with increased plasma-renin-angiotensin-aldosterone activity and elevated catecholamines, which results in vasoconstriction and further compromise to the gastric blood flow [11-14].

To our knowledge, this is the first large-scale national database analysis that looked at the incidence and predictors of GIB among hospitalized patients with ARDS. 
We conducted a study utilizing the National Inpatient Sample (NIS) database with the primary aim to determine the incidence of gastrointestinal hemorrhage among patients admitted with ARDS. As a secondary aim, we intended to analyze the comorbidities contributing to increased risk of GIB in those patients, and to evaluate the length of hospital stay, attributed health cost burden and mortality rates among ARDS patients with GIB versus those who did not develop GIB.

\section{Patients and Methods}

\section{Data source}

A retrospective longitudinal analysis of all adult patients ( $>$ 18 years) admitted in the USA from 2002 to 2012 with the diagnosis of ARDS was performed using the NIS database. The NIS database was developed by the Health Care Cost and Utilization Project (HCUP) and is the largest publicly available all-payer inpatient care database available in the USA. This database is known for its utility in studying trends and outcomes of various diseases and procedures [15-17], and it comprises stratified data collected from $20 \%$ of US community hospitals, in the form of de-identified information on individual hospitalizations that includes demographics, insurance status, comorbidities, admission status, discharge diagnoses, procedures, outcomes, length of stay (LOS) and cost of hospitalization.

\section{Study population}

Using the International Classification of Diseases, Ninth Revision, Clinical Modification (ICD-9-CM) codes, we identified all hospitalizations among adults $>18$ years of age, admitted with a primary or secondary diagnosis of ARDS, from 2002 through 2012. Hospitalizations with missing age, gender, admission and discharge diagnoses or mortality were excluded. We also collected variable etiologies of ARDS to include community-acquired pneumonia, aspiration pneumonia, sepsis or septic shock, pancreatitis and burns.

\section{Definition of variables}

The NIS variables were utilized to identify patients' age, gender and race. Age was divided into five categories: younger than 20 years, 20 - 39 years, 40 - 59 years, 60 - 79 years and older than 80 years. Race was divided into white, black, Hispanic, Asian or Pacific Islander, and Native American. A modified Charlson comorbidity index (CCI) was used to define the severity of the associated comorbid conditions. The scores ranged from 0 to 33 with higher scores corresponding to a greater burden of comorbid disease and vice versa. Hospital bed size and its location as urban or rural were also taken into account. Hospitals that upheld an Accreditation Council for Graduate Medical Education-accredited residency program, a membership of the Council of Teaching Hospi- tals, and/or had a full-time equivalent interns and residents to provide a patient ratio of 0.25 were considered as "teaching hospitals". The cost of each hospitalization was calculated by merging the data with cost to charge ratio from the HCUP database. This was standardized and adjusted for each year. The cost of each year was adjusted in reference to the 2016 US dollar value by using the consumer price index to account for inflation.

\section{Outcomes}

Our objectives were as follows: 1) the primary objective was to analyze the incidence of GIB in patients hospitalized with ARDS in a cohort group of individuals admitted between 2002 and $2012 ; 2$ ) the secondary objective was to investigate the independent predictors of GIB in patients with ARDS. We analyzed the following well-established comorbidities associated with GIB: alcoholism, past or current peptic ulcer disease (PUD), underlying liver disease, atrial fibrillation (AF), congestive heart failure (CHF), valvular heart disease, renal failure, anemia, past history of GIB, coagulation disorders, and gastrointestinal malignancies including esophageal, gastric, small intestinal, colorectal cancers [18]. Established risk factors for the development of ARDS like sepsis and septic shock, aspiration pneumonia and community-acquired pneumonia, burns and pancreatitis were also studied [19]. We also compared the LOS and cost per hospitalization of patients with ARDS and GIB versus those who did not have GIB. Mortality rates between the two groups were also assessed.

\section{Statistical analysis}

ARDS patients were captured by ICD-9 code 518.82 whether ARDS was a primary or a secondary diagnosis. GIB in those patients was also extracted using the corresponding clinical classification software codes. A Chi-square test or analysis of variance (ANOVA) was accordingly used to identify the demographical differences between ARDS patients with and without GIB. A two-sample $t$-test was used to assess for impact of GIB on in-hospital mortality, LOS and total charges for ARDS patients with or without GIB. A univariate logistic regression analysis was used to calculate odds ratios (OR) for causes of ARDS, comorbid conditions and their associations with the incidence of GIB. A Multivariate analysis of significant co-morbidities was then performed to account for any confounders among the significant comorbidities.

\section{Results}

A total of 149,190 patients (older than 18 years) were identified between 2002 and 2012 with a discharge diagnosis of ARDS. A total of 3,988 of those patients with ARDS developed GIB. The mortality rate during hospitalization was significantly higher in patients with ARDS complicated by GIB reaching to $27 \%$ when compared to $11 \%$ in patients without 
Table 1. Baseline Characteristics and Demographics of ARDS Patients With and Without GIB

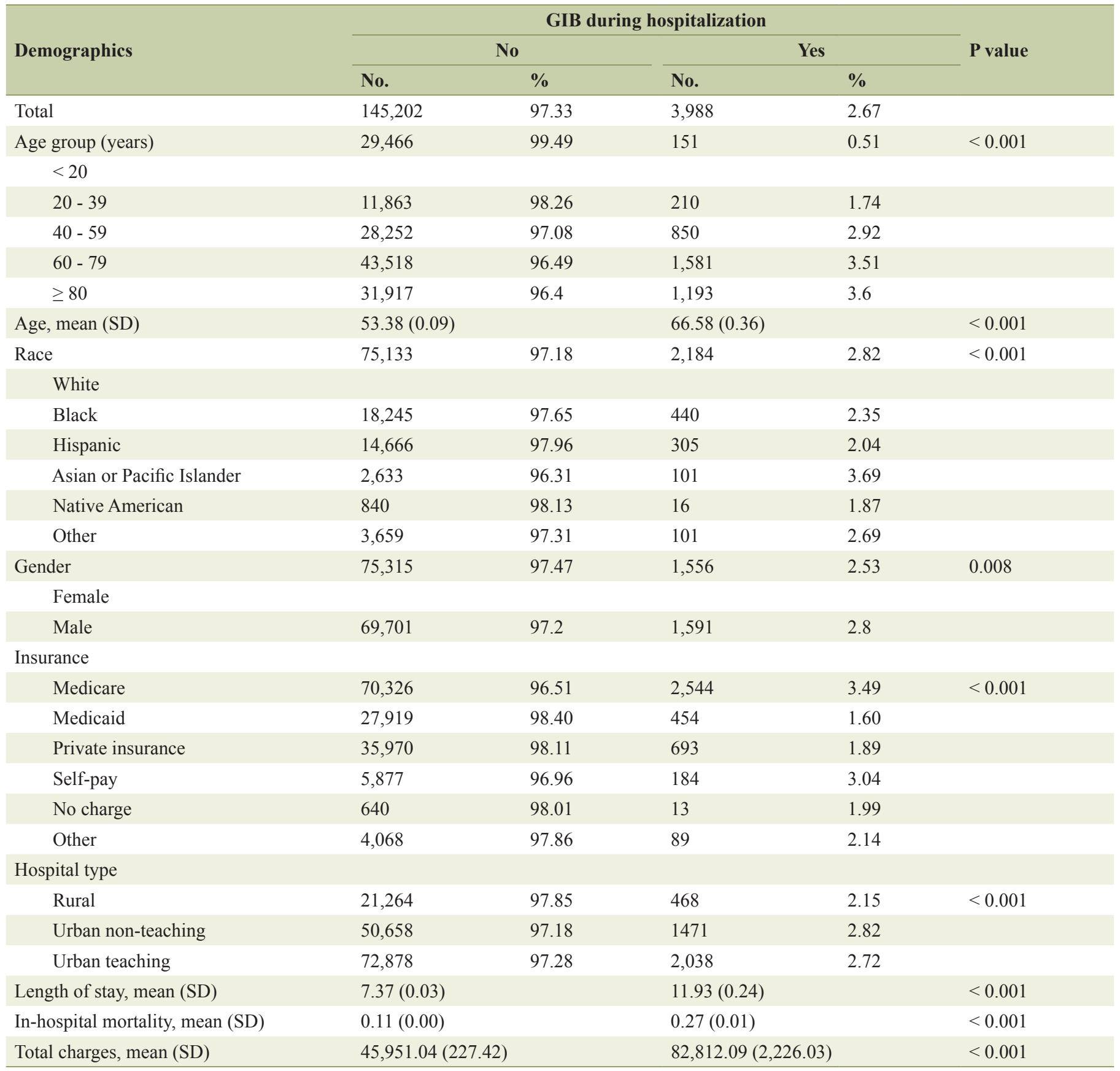

\section{GIB $(\mathrm{P}<0.001)$.}

Table 1 shows the baseline characteristics and demographics of patients with ARDS with and without GIB. The mean age of patients with ARDS who had GIB was 67 years, and more than $7 \%$ of these individuals were over 60 years of age. There was no significant gender difference among the patients who had GIB. Asians and Pacific Islanders exhibited a significantly higher risk of GIB when compared to other races (3.69\%; P < 0.001). Patients who had GIB were more likely to have Medicare as their medical coverage provider $(3.49 \%$;
$\mathrm{P}<0.001)$. Most ARDS patients with GIB had CCI index $>$ 2. Patients with ARDS who had GIB had significantly longer length of hospital stay of 11.93 days (SD: 0.24 ; $\mathrm{P}<0.001$ ) as compared to 7.37 days (SD: 0.03; $\mathrm{P}<0.001$ ) for patients who did not develop a GIB. The mean hospital cost was also inflated in patients with GIB as compared to patients without GIB $(\$ 82,812$ versus $\$ 45,951 ; \mathrm{P}<0.001)$.

Table 2 shows the various comorbidities that were associated with an increased risk of GIB. Patients with pre-existing gastrointestinal conditions like cirrhosis and peptic ulcer dis- 
Table 2. Risk Factors Associated With GIB in Patients With ARDS (CCl: Charlson Comorbidity Index)

\begin{tabular}{|c|c|c|c|c|}
\hline Comorbidity & Odds ratio & & nce interval & P value \\
\hline Gastroesophageal reflux disease & 0.754 & 0.669 & 0.85 & $<0.001$ \\
\hline Atrial fibrillation & 1.549 & 1.431 & 1.676 & $<0.001$ \\
\hline Peptic ulcer disease & 3.705 & 3.079 & 4.458 & $<0.001$ \\
\hline Rheumatoid arthritis & 0.789 & 0.571 & 1.09 & 0.1354 \\
\hline Hypertension & 1.048 & 0.983 & 1.118 & 0.1547 \\
\hline Chronic obstructive pulmonary disorder & 1.041 & 0.96 & 1.128 & 0.3415 \\
\hline Diabetes & 0.982 & 0.909 & 1.06 & 0.6351 \\
\hline Tobacco use disorder & 0.673 & 0.583 & 0.777 & $<0.001$ \\
\hline Anemia & 2.472 & 2.312 & 2.644 & $<0.001$ \\
\hline Peripheral vascular disease & 1.304 & 1.114 & 1.525 & 0.0009 \\
\hline TIA/stroke & 1.166 & 1.033 & 1.316 & 0.0126 \\
\hline Weekend admission & 1.035 & 0.962 & 1.114 & 0.3617 \\
\hline Coagulopathy & 3.003 & 2.738 & 3.294 & $<0.001$ \\
\hline Diverticular bleeding & 2.742 & 1.441 & 5.218 & $<0.001$ \\
\hline Esophageal cancer & 1.23 & 0.707 & 2.14 & 0.4628 \\
\hline Stomach cancer & 3.09 & 2.035 & 4.692 & $<0.001$ \\
\hline Colon cancer & 1.813 & 1.431 & 2.299 & $<0.001$ \\
\hline
\end{tabular}

ease had higher predisposition to develop GIB with an odds ratio (OR) of $8.4(95 \% \mathrm{CI}: 7.4-9.4)$ and $3.7(95 \% \mathrm{CI}: 1.4$ - 1.7), respectively. Patients with a history of gastric cancer, diverticular bleeding and colon cancer were more likely to have GIB. However, patients with gastroesophageal reflux disease (GERD) had a relatively lower OR (OR: 0.8; 95\% CI: 0.7 0.9 ) of having a GIB. Furthermore, patients with coagulopathy (OR: 3.003; P < 0.001), pancytopenia (OR: 2.6; P < 0.001) and anemia (OR: 2.5; P $<0.001$ ) had higher likelihood of developing GIB. Other conditions which raised the risk of GIB included $\mathrm{AF}$ and $\mathrm{CHF}$.

On the other hand, comorbidities like coronary artery disease (CAD), valvular heart disease, hypertension, COPD, diabetes, end-stage renal disease (ESRD), rheumatoid arthritis and tobacco use had a relatively lower incidence of GIB.

Table 3 shows the most common causes of ARDS and their attributed increased risk of GIB. These included aspiration pneumonia, pancreatitis and sepsis in decreasing order. ARDS secondary to burns had lower OR to have a GIB.

\section{Discussion}

Our study is the largest to date, where a nationwide analysis of the incidence of GIB in individuals admitted to intensive care units with ARDS, was performed. Data collected over a decade

Table 3. Common Etiologies of ARDS and Their Risk for GIB

\begin{tabular}{lllcl}
\hline Cause & Odds ratio & & $\mathbf{9 5 \%}$ confidence interval & Pvalue \\
\hline Aspiration pneumonia & 2.098 & 1.916 & 2.296 & $<0.001$ \\
Pancreatitis & 2.016 & 1.633 & 2.49 & $<0.001$ \\
Sepsis & 1.622 & 1.439 & 1.828 & $<0.001$ \\
Pneumonia & 0.833 & 0.776 & 0.894 & $<0.001$ \\
Burns & 0.385 & 0.096 & 1.552 & 0.1797 \\
\hline
\end{tabular}


using the NIS database were evaluated. Our findings could be summarized by the following: 1) there is an increased incidence of GIB among critically ill patients suffering ARDS particularly in elderly individuals; 2) the hospital mortality rate among ARDS patients with GIB was significantly increased compared to the control group who did not develop GIB; 3 ) predictors of GIB included advanced age, cirrhosis and peptic ulcer disease; 4) potential causes of ARDS that were statistically linked to an increased risk of GIB were aspiration pneumonia, pancreatitis and sepsis or septic shock; 5) in addition, there was a significant increase in overall length of hospital stay and attributed health care cost among patients whose course of stay was complicated by GIB.

The incidence of GIB among ARDS patients in our study reached around $2.67 \%$. This is comparable to a multicenter prospective trial by Krag et al, where the authors reported a GIB rate of $2.6 \%(95 \%$ CI: $1.6-3.6 \%)$ among mechanically ventilated critically ill patients [3]. Similar results with a prevalence ranging from $0.6 \%$ to $4 \%$ have been reported in the literature as well $[5,20]$. This is in contrast to the earlier reports in 1978 where overt GIB rates were thought to be as high as $25 \%$ in critically ill patients [21]. This difference could be explained in part by an overall improvement in the management of critically ill patients, ranging from early aggressive resuscitation, institution of early enteral nutrition, as well as increasing use of prophylactic acid suppression measures to prevent stress ulcer formation [22-24].

An acute or chronic underlying liver disease has been described in the literature by Barletta to be an independent risk factor for GIB among patients with severe sepsis and septic shock [25]. Our data comparably showed a significant increased risk of GIB among ARDS patients with liver cirrhosis (OR: 8.36 (95\% CI: $0.66-0.85) ; \mathrm{P}<0.0001)$ and a history of peptic ulcer disease (OR: 3.705; $\mathrm{P}<0.0001)$. Other potential risk factors that we were able to identify in our ARDS study group included coagulopathy, pancytopenia and anemia. Various studies in the past have demonstrated an independent correlation between GIB and coagulopathy or thrombocytopenia in critically ill patients $[3,5,26]$. Approximately $95 \%$ of all patients admitted to the intensive care unit are reported to exhibit a drop in their hemoglobin by their third day of stay [27].

Our study elucidated other comorbidities that were independently linked to a higher incidence of GIB like AF, CHF and history of diverticular bleeding or gastrointestinal malignancies. An earlier retrospective study reported in 2003 by Faisy et al analyzed the incidence of GIB in critically ill patients who received stress ulcer prophylaxis in comparison to patients who did not. The patients who developed clinically significant GIB were found to have a higher Simplified Acute Physiology Score (SAPS II) [22]. Similarly, another study by Krag et al demonstrated a higher Sequential Organ Failure Assessment (SOFA) score to be an independent risk factor for GIB in sick patients admitted to the critical care unit [3]. These studies further illustrate the attributed increased risk of GIB in critically ill patients with ARDS and multiple other associated comorbidities.

On another note, ARDS patients who receive positive pressure ventilation (PPV) for $>3$ days are in particular susceptible to mucosal damage due to splanchnic hypo-perfusion that furthermore worsens when the PEEP levels up to $15-20$ $\mathrm{cm}$ of $\mathrm{H}_{2} \mathrm{O}$ [28]. The surviving sepsis guidelines currently recommend stress ulcer prophylaxis with proton pump inhibitor (PPI), especially in mechanically ventilated patients for $>48$ $h$. This was endorsed despite the lack of high-level evidence showing any mortality benefit [29]. The lack of clear benefit with the use of acid suppressants could be hypothesized largely due to failure in distinguishing between SRMD- and non-SRMD-related bleeding, such as variceal bleeding, vascular anomalies or diverticular bleeding. Stress ulcer prophylaxis does not play a preventative role in the non-SMRD-related bleeding.

GERD, nasogastric tubes (NGTs) and duodenogastroesophageal bile reflux are potential proposed mechanisms of esophageal injury in critically ill patients [30]. Interestingly, our study did not show GERD to be a significant predictor of GIB among ARDS patients (OR: 0.754; P $<0.0001$ ). Wilmer et al who looked at ventilated patients also noted this interesting finding, and recognized that majority of esophageal mucosal injury $(75 \%)$ were secondary to bile reflux and direct NGT trauma [31].

Our study showed a substantially increased length of hospital stay and cost of care among patients with ARDS complicated by a GIB.

One of our major findings included a significant increase in hospital mortality from $11 \%$ to $27 \%$ among ARDS patients with GIB as compared to the ARDS group without GIB. The cause for this is likely to be multifactorial, including severity of comorbidities, organ failure and age. However, we cannot comment on the long-term outcomes among this population of patients after discharge.

The limitation of our study is that data were derived from an administrative database, which poses the possibility of incorrect classification due to coding errors. However, it is important to note that annual quality assessment of the NIS database is employed by the database administrators to maintain internal validity. Another limitation of our study is the lack of long-term data; as such, we can only assess the short-term inpatient outcomes and mortality. Moreover, as a retrospective observational study, no inferences can be made regarding causality between risk factors and outcomes. The observed risk factors are reported rather as associations.

\section{Conclusions}

In summary, our study is the first large-scale population-based study in the USA to examine the relationship between GIB and ARDS in critically ill patients. We concluded that the incidence of GIB among patients with ARDS is relatively high, reaching up to $2.67 \%$. Furthermore, the occurrence of GIB in patients with ARDS is associated with an increased mortality rate, longer hospitalizations and a considerable increase in health care cost. Heightened awareness is needed to consider early prophylaxis and prevention of this dreaded complication in susceptible patients with ARDS, specifically those who primarily had pancreatitis, pneumonia or septic shock. The main limitation of our study is related to our reliance on administrative data prepared for the purpose of billing rather than clinical 
care or research. Further studies, namely, prospective trials, are needed to shed more light on this subject.

\section{Conflict of Interest}

None.

\section{Financial Support}

No financial disclosures.

\section{Potential Competing Interests}

None.

\section{Guarantor of the Article}

Jobin Philipose accepted full responsibility for the conduct of the study.

\section{Author Note}

The abstract for the above manuscript was presented as a poster in the American College of Gastroenterology Meeting in Orlando on October 15, 2017. The poster was a recipient of Presidential Poster Award for the same meeting.

\section{References}

1. Gastrointestinal bleeding in acute respiratory failure. $\mathrm{Br}$ Med J. 1978;1(6112):531.

2. Ben-Menachem T, Fogel R, Patel RV, Touchette M, Zarowitz BJ, Hadzijahic N, Divine G, et al. Prophylaxis for stress-related gastric hemorrhage in the medical intensive care unit. A randomized, controlled, single-blind study. Ann Intern Med. 1994;121(8):568-575.

3. Krag M, Perner A, Wetterslev J, Wise MP, Borthwick M, Bendel S, McArthur C, et al. Prevalence and outcome of gastrointestinal bleeding and use of acid suppressants in acutely ill adult intensive care patients. Intensive Care Med. 2015;41(5):833-845.

4. Shuman RB, Schuster DP, Zuckerman GR. Prophylactic therapy for stress ulcer bleeding: a reappraisal. Ann Intern Med. 1987;106(4):562-567.

5. Cook DJ, Fuller HD, Guyatt GH, Marshall JC, Leasa D, Hall R, Winton TL, et al. Risk factors for gastrointestinal bleeding in critically ill patients. Canadian Critical Care Trials Group. N Engl J Med. 1994;330(6):377-381.

6. Plummer MP, Blaser AR, Deane AM. Stress ulceration: prevalence, pathology and association with adverse outcomes. Crit Care. 2014;18(2):213.

7. Schindlbeck NE, Lippert M, Heinrich C, Muller-Lissner
SA. Intragastric bile acid concentrations in critically ill, artificially ventilated patients. Am J Gastroenterol. 1989;84(6):624-628.

8. Lucas CE, Sugawa C, Riddle J, Rector F, Rosenberg B, Walt AJ. Natural history and surgical dilemma of "stress" gastric bleeding. Arch Surg. 1971;102(4):266-273.

9. Czaja AJ, McAlhany JC, Pruitt BA, Jr. Acute gastroduodenal disease after thermal injury. An endoscopic evaluation of incidence and natural history. N Engl J Med. 1974;291(18):925-929.

10. Peura DA, Johnson LF. Cimetidine for prevention and treatment of gastroduodenal mucosal lesions in patients in an intensive care unit. Ann Intern Med. 1985;103(2):173177.

11. Sellden H, Sjovall H, Ricksten SE. Sympathetic nerve activity and central haemodynamics during mechanical ventilation with positive end-expiratory pressure in rats. Acta Physiol Scand. 1986;127(1):51-60.

12. Tanaka S, Sagawa S, Miki K, Claybaugh JR, Shiraki K. Changes in muscle sympathetic nerve activity and renal function during positive-pressure breathing in humans. Am J Physiol. 1994;266(4 Pt 2):R1220-1228.

13. Chernow B, Soldano S, Cook D, Lyons P, Barton M, Casey LC, Fletcher JR, et al. Positive end-expiratory pressure increases plasma catecholamine levels in non-volume loaded dogs. Anaesth Intensive Care. 1986;14(4):421425.

14. Aneman A, Ponten J, Fandriks L, Eisenhofer G, Friberg P, Biber B. Hemodynamic, sympathetic and angiotensin II responses to PEEP ventilation before and during administration of isoflurane. Acta Anaesthesiol Scand. 1997;41(1 Pt 1):41-48.

15. Ahmed M, Kanotra R, Savani GT, Kotadiya F, Patel N, Tareen S, Fasullo MJ, et al. Utilization trends in inpatient endoscopic retrograde cholangiopancreatography (ERCP): A cross-sectional US experience. Endosc Int Open. 2017;5(4):E261-E271.

16. Kanotra R, Ahmed M, Patel N, Thakkar B, Solanki $\mathrm{S}$, Tareen S, Fasullo MJ, et al. Seasonal variations and trends in hospitalization for peptic ulcer disease in the United States: a 12-year analysis of the nationwide inpatient sample. Cureus. 2016;8(10):e854.

17. Badheka AO, Patel NJ, Singh V, Shah N, Chothani A, Mehta K, Deshmukh A, et al. Percutaneous aortic balloon valvotomy in the United States: a 13-year perspective. Am J Med. 2014;127(8):744-753 e743.

18. Gibson JA, Odze RD. Pathology of diseases that cause upper gastrointestinal tract bleeding. Gastrointest Endosc Clin N Am. 2011;21(4):583-596.

19. Pham T, Rubenfeld GD. Fifty years of research in ARDS. The epidemiology of acute respiratory distress syndrome. A 50th birthday review. Am J Respir Crit Care Med. 2017;195(7):860-870.

20. Alhazzani W, Alenezi F, Jaeschke RZ, Moayyedi P, Cook DJ. Proton pump inhibitors versus histamine 2 receptor antagonists for stress ulcer prophylaxis in critically ill patients: a systematic review and meta-analysis. Crit Care Med. 2013;41(3):693-705.

21. Hastings PR, Skillman JJ, Bushnell LS, Silen W. Antacid 
titration in the prevention of acute gastrointestinal bleeding: a controlled, randomized trial in 100 critically ill patients. N Engl J Med. 1978;298(19):1041-1045.

22. Faisy C, Guerot E, Diehl JL, Iftimovici E, Fagon JY. Clinically significant gastrointestinal bleeding in critically ill patients with and without stress-ulcer prophylaxis. Intensive Care Med. 2003;29(8):1306-1313.

23. El-Kersh K, Jalil B, McClave SA, Cavallazzi R, Guardiola J, Guilkey K, Persaud AK, et al. Enteral nutrition as stress ulcer prophylaxis in critically ill patients: A randomized controlled exploratory study. J Crit Care. 2018;43:108-113.

24. Barletta JF, Kanji S, MacLaren R, Lat I, Erstad BL, American-Canadian consortium for Intensive care Drug utilization I. Pharmacoepidemiology of stress ulcer prophylaxis in the United States and Canada. J Crit Care. 2014;29(6):955-960.

25. Barletta JF. Histamine-2-receptor antagonist administration and gastrointestinal bleeding when used for stress ulcer prophylaxis in patients with severe sepsis or septic shock. Ann Pharmacother. 2014;48(10):1276-1281.

26. Strauss R, Wehler M, Mehler K, Kreutzer D, Koebnick C, Hahn EG. Thrombocytopenia in patients in the medical intensive care unit: bleeding prevalence, transfusion requirements, and outcome. Crit Care Med. 2002;30(8):1765-1771.

27. Corwin HL, Krantz SB. Anemia of the critically ill: "acute" anemia of chronic disease. Crit Care Med. 2000;28(8):3098-3099.

28. Holland A, Thuemer O, Schelenz C, van Hout N, Sakka SG. Positive end-expiratory pressure does not affect indocyanine green plasma disappearance rate or gastric mucosal perfusion after cardiac surgery. Eur J Anaesthesiol. 2007;24(2):141-147.

29. Rhodes A, Evans LE, Alhazzani W, Levy MM, Antonelli M, Ferrer R, Kumar A, et al. Surviving Sepsis Campaign: International Guidelines for Management of Sepsis and Septic Shock: 2016. Crit Care Med. 2017;45(3):486-552.

30. Plaisier PW, van Buuren HR, Bruining HA. An analysis of upper GI endoscopy done for patients in surgical intensive care: high incidence of, and morbidity from reflux oesophagitis. Eur J Surg. 1997;163(12):903-907.

31. Wilmer A, Tack J, Frans E, Dits H, Vanderschueren S, Gevers A, Bobbaers H. Duodenogastroesophageal reflux and esophageal mucosal injury in mechanically ventilated patients. Gastroenterology. 1999;116(6):1293-1299. 